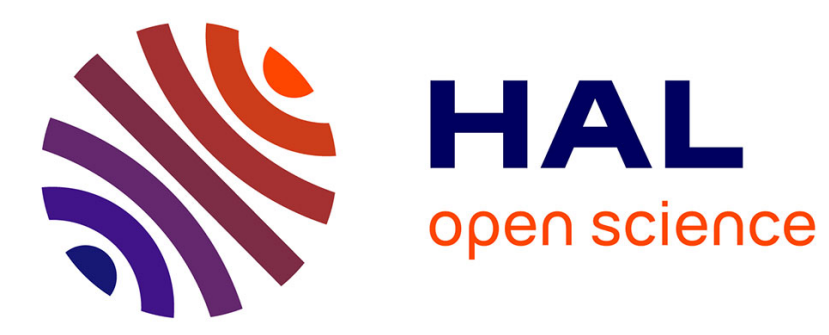

\title{
Characterizations of Solutions in Geochemistry: Existence, Uniqueness and Precipitation Diagram
}

\author{
Jocelyne Erhel, Tangi Migot
}

\section{To cite this version:}

Jocelyne Erhel, Tangi Migot. Characterizations of Solutions in Geochemistry: Existence, Uniqueness and Precipitation Diagram. Computational Geosciences, 2019, 23 (3), pp.523-535. 10.1007/s10596018-9800-2 . hal-01584490v2

\section{HAL Id: hal-01584490 \\ https://hal.inria.fr/hal-01584490v2}

Submitted on 4 Dec 2018

HAL is a multi-disciplinary open access archive for the deposit and dissemination of scientific research documents, whether they are published or not. The documents may come from teaching and research institutions in France or abroad, or from public or private research centers.
L'archive ouverte pluridisciplinaire HAL, est destinée au dépôt et à la diffusion de documents scientifiques de niveau recherche, publiés ou non, émanant des établissements d'enseignement et de recherche français ou étrangers, des laboratoires publics ou privés. 


\title{
Characterizations of Solutions in Geochemistry: Existence, Uniqueness and Precipitation Diagram
}

\author{
Jocelyne Erhel · Tangi Migot
}

Received: date / Accepted: date

\begin{abstract}
In this paper, we study the properties of a geochemical model involving aqueous and precipitationdissolution reactions at a local equilibrium in a diluted solution. This model can be derived from the minimization of the free Gibbs energy subject to linear constraints. By using logarithmic variables, we define another minimization problem subject to different linear constraints with reduced size. The new objective function is strictly convex, so that uniqueness is straightforward. Moreover, existence conditions are directly related to the totals, which are the parameters in the mass balance equation. These results allow us to define a partition of the totals into mineral states, where a given subset of minerals are present. This precipitation diagram is inspired from thermodynamic diagrams where a phase depends on physical parameters. Using the polynomial structure of the problem, we provide characterizations and an algorithm to compute the precipitation diagram. Numerical computations on some examples illustrate this approach.
\end{abstract}

Keywords geochemistry · existence · uniqueness · precipitation diagram · modeling · polynomial system . convex optimization $\cdot$ precipitation-dissolution reaction

J. Erhel

INRIA Rennes

E-mail: jocelyne.erhel@inria.fr

T. Migot

IRMAR-INSA de Rennes

E-mail: tangi.migot@insa-rennes.fr

\section{Introduction}

Many environmental studies rely on modeling geochemical reactions such as aquifer contamination, underground waste disposal, remediation. In some of these applications the reactions can be assumed to be fast compared with their environment and so the reactions are considered in a local equilibrium. In this latter case, the model involves non-linear algebraic equations [2]. Here, we focus on aqueous and precipitation-dissolution reactions so only liquid and mineral species are involved. Many applications consider geochemical reactions coupled with hydrodynamic processes as in reactive transport model [26]. It is therefore of great interest to get a deep knowledge of the geochemical properties and to solve efficiently the algebraic equations.

We consider here a diluted solution, as in some reactive transport models such as $[21,22]$ or some exercises such as the MoMaS benchmark [8,9]. We use an approach based on the definition of primary and secondary aqueous species, which are related by mass action laws [39]. Regarding the minerals, their appearance or disappearance is governed by saturation thresholds. The mathematical model is completed by mass balance equations, with so-called totals which are given data of the system, as for example in [15,25].

In order to solve the non-linear equations resulting from the geochemical model, the main challenge is to find out which minerals are precipitated and which ones are dissolved, in other words to check whether saturation thresholds are active or not. Some numerical algorithms are based on a combinatorial approach $[1,3,31]$. Because the system can be formulated as a non-linear complementarity problem [12], numerical algorithms are frequently based on semi-smooth Newton methods [19,22] or interior-point methods [24,33,34]. 
Complementarity problems have been extremely popular in the field of optimization theory and have been extensively studied, [16] and references therein. Many first-order optimality conditions of a minimization problem with inequality constraints can be cast as a complementarity problem. In the other way round, the geochemical model can be viewed as first-order optimality conditions.

This approach was previously used connected with the minimization of the Gibbs energy, see for instance in $[36,37]$. More recent theoretical studies also took this path in the context of reactive transport in $[5,21,25]$. The Gibbs energy is a function of all the species (primary and secondary aqueous species, minerals). Linear constraints are the mass balance equations (dimension the number of aqueous components) and positive quantities of minerals (dimension the number of minerals). Minimizing the Gibbs energy with these constraints is a convex problem, ensuring a global minimum. However, it requires additional assumptions or transformations to get existence or uniqueness, see for instance $[5,36]$.

In this paper, we propose a different formulation, which is new as far as we know. The objective function is based on the mass balance equation and the mass action laws, whereas linear constraints are conditions on the saturation thresholds. Under mild assumptions on the stoichiometry, we can prove that the first-order optimality conditions are nothing else than the geochemical model considered. This minimization problem has several merits compared to the aforementioned one. The variables of the objective function are only the aqueous components and the number of constraints is only the number of minerals. Moreover, the objective function is strictly convex, so that it is easy to assess the uniqueness of a solution. The analysis of existence is directly related to the totals, which are input data of the model. Finally, the strict convexity as well as the reduced dimensions would allow interesting numerical performances.

In order to do so, we have to use logarithmic variables for the aqueous species, thus to assume that the variables are strictly positive. This usage of logarithms is quite common in the literature, see for example [7, $14,20,27,35]$. In this paper, we carefully analyze the mathematical model and prove that it is equivalent to a reduced system with only strictly positive aqueous variables, completed by an independent trivial system with non negative aqueous variables. To the best of our knowledge, our theoretical justification of logarithms is different from the literature, as in $[5,36]$.

As we already pointed out, one motivation is to characterize the set of precipitated minerals and the complement set of dissolved minerals. First, we charac- terize the set of data $\mathcal{T}$ such that there exists a solution. Therefore, we can associate the totals in $\mathcal{T}$ to a unique geochemical system. Then, we introduce the concept of precipitation diagram. More precisely, we build a partition of the set $\mathcal{T}$ such that each subset is associated to a geochemical system with a given set of precipitated minerals. This partition defines a diagram, in the same spirit as thermodynamic diagrams (or stability diagrams), see for instance $[11,13]$. However, in our case, the axis are the total concentrations and not some physical quantities like the pressure or the temperature.

We propose to build precipitation diagrams thanks to symbolic computations. This approach provides a graphical view of the conditions under which some minerals can precipitate. With numerical values of given totals, the symbolic approach can compute the solution, although this is not the main objective. Moreover, the diagram can be used in the context of reactive transport models, for example to provide an initial guess of non-linear algorithms or to find out in which subset are the totals. Then the complementary problem could be replaced by an easier system of equations, which can be solved by a classical Newton's method.

We developed a software in Maple and used it for several test cases. These experiments show that precipitation diagrams are quite complex, even for simple examples. Thus, it would not be easy to compute them numerically. We also point out that the CPU and memory requirements are reasonable.

The paper is organized as follows: in Section 2, we describe the geochemical model and show that it can be reduced to equations with strictly positive variables, justifying the use of logarithmic unknowns. We introduce an elementary example of geochemical systems as a common thread throughout the paper. Then, in Section 3 , we define the equivalent optimization problem and prove uniqueness of the solution along with some results of existence. We provide an example where existence is conditioned by the totals. Based on these theoretical outputs, we introduce in Section 4 our concept of a precipitation diagram. Our numerical experiments of Section 5 illustrate the complexity of these diagrams and the information they provide. Finally, we discuss some future work in Section 6 .

\section{A Model for Geochemistry}

In this section, we describe a mathematical model for geochemistry, which has already been used in the context of reactive transport, see $[14,15,35]$. We consider a species/components model frequently used in geochemistry modeling as in [2] or [38]. 
We consider here aqueous and precipitation-dissolution So, there are two possible cases, either the fluid is underreactions, so that species can be in solution or minerals. For the sake of simplicity, we do not include reactions with fixed species other than minerals.

We first describe the mathematical model, then we show that we can consider aqueous species with positive concentrations. Therefore we can introduce an equivalent model with logarithms.

\subsection{Mass action laws and mass balance equations}

We consider here a diluted aqueous solution, so that the activity of water is equal to 1 and the activity of an aqueous species is equal to its molar concentration. We also assume that a precipitation-dissolution reaction involves only one mineral.

A model of this type is proposed in the MoMaS benchmark $[8,9]$. These assumptions are also done in some numerical models of reactive transport [21,22].

Aqueous species can be split through a Morel table [30]. Variables of the problem are then the concentrations of the primary aqueous components $c \in \mathbb{R}_{+}^{N_{c}}$, those of the secondary aqueous species $\alpha \in \mathbb{R}_{+}^{N_{\alpha}}$, and the quantities of minerals $p \in \mathbb{R}_{+}^{N_{p}}$. All of them are non-negative quantities.

Without loss of generality, the stoichiometric coefficients can be chosen as integers. Let $S \in \mathbb{Z}^{N_{\alpha} \times N_{c}}$ be the stoichiometric matrix for aqueous reactions associated with the positive constants of reaction $\kappa_{\alpha} \in \mathbb{R}^{N_{\alpha}}, \kappa_{\alpha}>$ 0 .

The law of mass action is directly used to model chemical equilibrium [25]. Here, because aqueous species can vanish, we write the law of mass action in a product form $[35,36]$ :

$$
\alpha_{i}(c)=\kappa_{\alpha i} \prod_{k \in\left\{1, \ldots, N_{c}: S_{i k} \neq 0\right\}} c_{k}^{S_{i k}}, i=1, \ldots, N_{\alpha} .
$$

Remark 1 Since for all $k, c_{k} \geq 0$, then for all $i, \alpha_{i} \geq 0$. Moreover, if, for some $i S_{i k}<0$, then the law of mass action requires $c_{k}>0$.

Precipitation-dissolution reactions cannot be treated in the same way, since the activity of a mineral is constant. These are heterogeneous reactions, which are represented with a matrix $E \in \mathbb{Z}^{N_{p} \times N_{c}}$ of integer stoichiometric coefficients and a vector of solubility products denoted by $\kappa_{p} \in \mathbb{R}^{N_{p}}$ with $\kappa_{p}>0$. Appearance and disappearance of the mineral $j, j=1, \ldots, N_{p}$, are governed by a saturation threshold $\gamma_{j}(c)$ defined by

$$
\gamma_{j}(c)=\prod_{k \in\left\{1, \ldots, N_{c}: E_{j k} \neq 0\right\}} c_{k}^{E_{j k}}, j=1, \ldots, N_{p} .
$$
saturated and there is no mineral,

$$
p_{j}=0, \kappa_{p j}-\gamma_{j}(c)>0, j=1, \ldots, N_{p},
$$

or the fluid is saturated and the mineral is present,

$$
p_{j} \geq 0, \kappa_{p j}-\gamma_{j}(c)=0, j=1, \ldots, N_{p}
$$

By convention we use a strict inequality in the undersaturated case to avoid any confusion. This kind of equilibrium problem is well-known in optimization theory under the name of complementarity problem, [12]. This problem may be reformulated as

$$
p \geq 0, \kappa_{p}-\gamma(c) \geq 0, p^{T}\left(\kappa_{p}-\gamma(c)\right)=0 .
$$

The model is completed by a mass balance equation, written for each primary aqueous component. Let $T \in$ $\mathbb{R}^{N_{c}}$ be the vector of total concentrations, which is given data in the context of a closed geochemical system. The mass balance equation is then written

$$
T=c+S^{T} \alpha(c)+E^{T} p .
$$

Finally, combining these three equations gives the geochemical model:

$\left\{\begin{array}{l}c+S^{T} \alpha(c)+E^{T} p-T=0 \\ p^{T}\left(\kappa_{p}-\gamma(c)\right)=0 \\ p \geq 0, \kappa_{p} \geq \gamma(c) \\ c \geq 0, \text { and } c_{k}>0 \forall k \in \mathcal{Z},\end{array}\right.$

with $\mathcal{Z}=\left\{k: 1 \leq k \leq N_{c}, \exists i S_{i k}<0\right.$ or $\left.\exists j E_{j k}<0\right\}$.

This system is a closed system of $\left(N_{c}+N_{p}\right)$ equations with inequality constraints. With our assumptions the coefficients are integer, so that all the expressions are polynomials. This observation will be used in Section 4.3.

The Gibbs energy $G(c, \alpha, p)$ is defined by chemical potentials for $c \geq 0, \alpha \geq 0, p \geq 0$. The usual gradient of $G$ uses logarithms and requires $c>0, \alpha>0$ [36]. If $G$ is defined in the domain $c>0, \alpha>0$, then equations (1), where the constraint $c \geq 0$ is replaced by $c>0$, are nothing else than the KKT conditions applied to the minimization of the Gibbs energy, $G(c, \alpha, p)$, subject to the mass conservation law and the constraint $p \geq 0$ [24]. Since $G$ is convex and the constraints are linear, the KKT conditions are necessary and sufficient provided that the constraints are feasible.

\subsection{A logarithmic model}

In order to use $\log (c)$, we have to study systems where an aqueous species could vanish. We first get rid of the particular case of a tracer, which appears for example in the MoMaS benchmark. 
When a stoichiometric coefficient $S_{i k}$ is zero, the component $c_{k}$ does not participate in the reaction involving the secondary species $\alpha_{i}(c)$. Similarly, it does not participate in the reaction with the mineral $p_{j}$ if $E_{j k}=0$. A particular case arises when all the coefficients associated to a component are zero, which does not react with any other species. Such a component is called a tracer.

Proposition 1 Let $c_{k} \geq 0$ be an aqueous tracer: $\forall i, S_{i k}=$ 0 and $\forall j, E_{j k}=0$. If the $k^{\text {th }}$ mass balance equation is satisfied, then $T_{k} \geq 0$ and $c_{k}=T_{k}$.

Proof The $k^{\text {th }}$ mass balance equation is reduced to $T_{k}=$ $c_{k}$ and $c_{k} \geq 0$.

We already noticed in Remark 1 and in (1) that if at least one stoichiometric coefficient $S_{i k}$ or $E_{j k}$ is negative, then $c_{k}$ must be positive.

We now consider the case where all coefficients $S_{i k}$ and $E_{i k}$ of a component $c_{k}$ are non-negative. If the total $T_{k}$ of a component $c_{k}$ is zero, then this component is absent, as well as the secondary species and minerals associated. Such cases appear in the MoMaS benchmark.

Proposition 2 We assume that $\forall i, S_{i k} \geq 0, \forall j, E_{j k} \geq$ 0 and that the $k^{\text {th }}$ mass balance equation is satisfied. Then, $T_{k} \geq 0$. Moreover, if $T_{k}=0$, then $c_{k}=0$, and $S_{i k} \alpha_{i}(c)=0, i=1, \ldots, N_{\alpha}, E_{j k} p_{j}=0, j=1, \ldots, N_{p}$.

Proof By definition, the $k^{\text {th }}$ mass balance equation is written

$$
T_{k}=c_{k}+\sum_{i \in\left\{1, \ldots, N_{c}: S_{i k} \neq 0\right\}} S_{i k} \alpha_{i}(c)+\sum_{j \in\left\{1, \ldots, N_{c}: E_{j k} \neq 0\right\}} E_{j k} p_{j} .
$$

The conclusions follow as the right-hand side is a sum of non-negative terms.

Remark 2 With the assumptions of Proposition 2, the model has no solution if $T_{k}<0$.

Proposition 3 We assume that $\forall i, S_{i k} \geq 0, \forall j, E_{j k} \geq$ 0 . We also assume that $(c, p)$ is solution of (1). If $T_{k}>$ 0 , then $c_{k}>0$.

Proof Note that $(c, p)$ solution of (1) implies that the $k^{\text {th }}$ mass balance equation is satisfied and so $T_{k} \geq 0$ by the previous proposition.

We prove that if $c_{k}=0$, then $T_{k}=0$.

First, we notice that $c_{k}=0$ implies $\forall i, S_{i k} \alpha_{i}(c)=0$ and $\forall j, E_{j k} \gamma_{j}(c)=0$. Now, if $E_{j k}>0$, then $\gamma_{j}(c)=$ $0<\kappa_{p j}$ thus $p_{j}=0$ by the complementarity condition in (1). Finally, $\forall j, E_{j k} p_{j}=0$, thus $T_{k}=0$.
Therefore, any tracer and any component associated to non-negative coefficients with a null total can be removed from the system. Moreover, the secondary species or minerals associated with this component can also be removed from the model. Indeed, the reactions involving a primary component with a zero concentration can be removed from the system.

All in all, it is possible to define a reduced model where all the concentrations $c_{k}$ of the primary components are positive.

Proposition 4 Model (1) is equivalent to a reduced model and a trivial model, where in the reduced model:

- There is no tracer: $\forall k, \exists i, S_{i k} \neq 0$ or $E_{i k} \neq 0$.

- All the components are reactive: $\forall k, c_{k}>0$.

- All the secondary species are reactive: $\forall i, \alpha_{i}(c)>0$.

- All the saturation thresholds are positive: $\forall i, \gamma_{i}(c)>0$.

and, in the trivial model:

- For a tracer $k: c_{k}=T_{k}$.

- For a component $k$ with $\forall i, S_{i k} \geq 0, \forall j, E_{j k} \geq 0$ and $T_{k}=0: c_{k}=0$ and $\forall i, S_{i k} \alpha_{i}(c)=0, \forall j, E_{j k} p_{j}=0$.

Proof The result follows by combining Remark 1 with propositions 1,2 and 3 .

From now on, we consider such a reduced model, where the number of primary components is still denoted by $N_{c}$ and the positivity constraint is now $c>0$

$\left\{\begin{array}{l}c+S^{T} \alpha(c)+E^{T} p-T=0, \\ p^{T}\left(\kappa_{p}-\gamma(c)\right)=0, \\ c>0, p \geq 0, \kappa_{p} \geq \gamma(c) .\end{array}\right.$

Finally, we can introduce logarithmic variables and define an equivalent model. In this result and through the rest of the paper the logarithm and exponential of a vector are taken componentwise, that is $\log (z)=$ $\left(\log \left(z_{i}\right)\right)_{1 \leq i \leq n}$ and $\exp (z)=\left(\exp \left(z_{i}\right)\right)_{1 \leq i \leq n}$ for all $z \in$ $\mathbb{R}^{n}$.

Proposition 5 Model (2) is equivalent to the complementarity problem

$\left\{\begin{array}{l}\exp (x)+S^{T} \exp \left(\log \left(\kappa_{\alpha}\right)+S x\right)+E^{T} p-T=0, \\ p^{T}\left(E x-\log \left(\kappa_{p}\right)\right)=0, \\ p \geq 0, E x \leq \log \left(\kappa_{p}\right),\end{array}\right.$

where $x=\log (c)$.

It can be noted that some conditions on $T$ are necessary to ensure the existence of a solution.

Corollary 1 If a primary component $k$ has only non negative stoichiometric coefficients and if $T_{k} \leq 0$, then system (2) has no solution. 
Proof If the stoichiometric coefficients associated to the primary component $k$ are non-negative, the corresponding total mass $\left(\exp (x)+S^{T} \exp \left(\log \left(\kappa_{\alpha}\right)+S x\right)+E^{T} p\right)_{k}$ is strictly positive. Thus, if there exists a solution, the total $T_{k}$ satisfies $T_{k}>0$.

2.3 Example with two salts: sodium chloride and potassium chloride

We conclude this section by giving a first example of a geochemical system in order to illustrate and highlight some characteristics. Chemical reactions are only precipitation-dissolution in order to simplify the presentation. Therefore, there are as many reactions as minerals and the system is defined by the stoichiometric matrix $E$ and the solubility products $\kappa_{p}$.

This first example, with two salts, considers precipitation of sodium chloride $\mathrm{NaCl}$ and potassium chloride $\mathrm{KCl}$, involving the three aqueous components, sodium ion $\mathrm{Na}^{+}$, potassium ion $\mathrm{K}^{+}$and chloride ion $\mathrm{Cl}^{-}$. The two reactions, along with the solubility products, are summed up in the following table:

\begin{tabular}{|c|c|c|}
\hline Mineral & Reaction & $\kappa_{p}$ \\
\hline $\mathrm{NaCl}$ & $\mathrm{NaCl} \rightleftarrows \mathrm{Na}^{+}+\mathrm{Cl}^{-}$ & 37.5837 \\
\hline $\mathrm{KCl}$ & $\mathrm{KCl} \rightleftarrows \mathrm{K}^{+}+\mathrm{Cl}^{-}$ & 7.6208 \\
\hline
\end{tabular}

The stoichiometric matrix of this system is:

$$
E=\left(\begin{array}{lll}
1 & 0 & 1 \\
0 & 1 & 1
\end{array}\right)
$$

which is of full rank $N_{p}$.

Since all the coefficients are positive, the data $T$ must satisfy $T>0$.

\section{Equivalence with an Optimization Problem}

We already noticed that system (1) is the first-order optimality condition for minimizing the Gibbs energy subject to the mass balance law and non-negativity of the mineral quantities.

In this section, we prove that the geochemical system (3) is the first-order optimality condition of another minimization problem, with an objective function and inequality constraints of reduced dimensions. This result is the keystone for studying existence and uniqueness of solutions of the geochemical model. Furthermore, this study is directly related to the totals $T$, which are the parameters of the mass balance law. Another approach could use variational analysis tools, for instance from [16].

\subsection{Objective function and inequality constraints}

We first define the objective function of the optimization problem for $x \in \mathbb{R}^{N_{c}}$ by

$f(x)=e_{N_{c}}^{T} \exp (x)+e_{N_{\alpha}}^{T} \exp \left(\log \left(\kappa_{\alpha}\right)+S x\right)-T^{T} x,(4)$

where $e_{n}$ is the vector of dimension $n$ whose components are all ones.

The function $f$ is $C^{\infty}$ in $\mathbb{R}^{N_{c}}$ with a gradient given by

$$
\nabla f(x)=\exp (x)+S^{T} \exp \left(\log \left(\kappa_{\alpha}\right)+S x\right)-T,
$$

and a Hessian matrix given by

$\nabla^{2} f(x)=\mathcal{D}(\exp (x))+S^{T} \mathcal{D}\left(\exp \left(\log \left(\kappa_{\alpha}\right)+S x\right)\right) S,(5)$

where $\mathcal{D}(y)$ denotes a diagonal matrix with the elements of $y$ on the diagonal.

Then, we define inequality constraints by denoting the feasible set:

$$
\mathcal{F}=\left\{x \in \mathbb{R}^{N_{c}}, g(x) \leq 0\right\},
$$

where $g(x)=E x-\log \left(\kappa_{p}\right)$. Clearly, the feasible set is convex.

A typical assumption that we will use in the sequel to guarantee non-emptiness of $\mathcal{F}$ is to require $E$ to be a full rank matrix.

Lemma 1 Assume that $E$ is of full rank. Then, $\mathcal{F}$ is non-empty.

Proof For $E$ a full rank matrix, there exists a solution of the equation $E x=\log \left(\kappa_{p}\right)$.

Finally, we introduce the convex minimization problem

$\min _{x \in \mathcal{F}} f(x)$.

Remark 3 The objective function $f$ is based on mass balance and constraints are saturation limits, so that problem (6) has $N_{c}$ unknowns and $N_{p}$ linear constraints. On the other hand, minimizing the Gibbs energy $G$ and satisfying mass balance and positivity of minerals involves generally $\left(N_{c}+N_{\alpha}+N_{p}\right)$ variables and $\left(N_{c}+N_{p}\right)$ linear constraints. Reduced dimensions would be useful in numerical solving algorithms.

\subsection{Uniqueness of a solution}

We are now able to prove the equivalence between the model (3) and the minimization problem (6). Indeed, the complementarity problem is nothing else than the Karush-Kuhn-Tucker (KKT) optimality conditions [4]. Uniqueness of a solution immediately follows.

We first show that the objective function is strictly convex: 
Lemma 2 The Hessian matrix of $f$, denoted $\nabla^{2} f(x)$, is symmetric positive definite for any $x$ and the function $f$ is strictly convex in $\mathbb{R}^{N_{c}}$.

Proof The elements of the diagonal matrices in the Hessian matrix given in (5) are positive. Any matrix of the form $S^{T} D S$, where $D$ is a diagonal matrix with positive elements, is symmetric positive semi-definite, while the diagonal matrix $\exp (x)$ is symmetric positive definite (spd). Thus, for all $x$, the matrix $\nabla^{2} f(x)$ is also spd.

The strict convexity of $f$ follows as a consequence.

Remark 4 It can be shown, with some assumptions or a change of variables, that the Gibbs energy is also strictly convex, see for example $[36,5,21]$. Here, the strict convexity of $f$ is straightforward.

From this lemma, and using the linearity of the constraints, it becomes easy to prove that the equations (3) are the KKT conditions of the minimization problem (6) and to prove the uniqueness of a solution.

Theorem 1 Assume that the feasible set is non-empty, i.e. $\mathcal{F} \neq \emptyset$. Then, equations (3) are equivalent to solving the minimization problem (6). Moreover, if the minimization problem has a solution, it is unique.

Proof We first note that any $x \in \mathcal{F}$ satisfies a linear constraint qualification. Since the objective function $f$ is convex, the feasible set is non-empty convex and a linear constraint qualification is satisfied at any feasible point, the KKT conditions are necessary and sufficient. Using the gradient of $f$ and the Jacobian $E^{T}$ of $g$, it appears that these KKT conditions are exactly equations (3). Thus, $x$ is a solution of (6) if and only if there exists a Lagrange multiplier $p \in \mathbb{R}^{N_{p}}$ such that $x$ and $p$ are solutions of (3).

Uniqueness of $x$ is ensured by the strict convexity of $f,[23]$, proved in Lemma 2.

If we further assume that the matrix $E$ is of rank $N_{p}$, the Lagrange multiplier is also unique.

Corollary 2 Assume that the matrix $E$ is of rank $N_{p}$. If the minimization problem has a solution, then it is unique and the Lagrange multiplier $p$ is unique.

Proof Note that the feasible set is non-empty here thanks to Lemma 1.

The gradients of the inequality constraints are the lines of the matrix $E$ and are linearly independent if $E$ is of rank $N_{p}$. Thus, any $x \in \mathcal{F}$ satisfies a linear independence constraint qualification (LICQ), so that if there is a solution, then the Lagrange multiplier $p$ is unique. [23].
Consequently, Corollary 2 guarantees uniqueness of the solution of the geochemical system (3), whenever such solution exists.

\subsection{Existence of a solution}

In Corollary 1, we have shown that, with non-negative stoichiometric coefficients, the existence of a solution requires that $T>0$. Now, we prove that, for any full rank stoichiometric matrix $E$, there exists a solution of (6), provided that $T>0$.

Theorem 2 Assume that the matrix $E$ is of rank $N_{p}$, and that $T>0$. Then, the geochemical model (2) has a unique solution.

Proof Let $F(x)=e_{N_{c}}^{T} \exp (x)-T^{T} x, x \in \mathbb{R}^{N_{c}}$, clearly $\forall x \in \mathbb{R}^{N_{c}}, f(x)>F(x)$.

Now, $\forall k=1, \ldots, N_{c}, \lim _{x_{k} \rightarrow+\infty}\left(\exp \left(x_{k}\right)-T_{k} x_{k}\right)=$ $+\infty$; since, by assumption $T_{k}>0$, we also have $\forall k=$ $1, \ldots, N_{c}, \lim _{x_{k} \rightarrow-\infty}\left(\exp \left(x_{k}\right)-T_{k} x_{k}\right)=+\infty$. Thus the function $F$ is coercive, in other words

$\lim _{\|x\| \rightarrow+\infty} F(x)=+\infty$, and it follows that the function $f$ is also coercive.

This property ensures the existence of a solution of the minimization problem, since (6) is a problem of minimizing a continuous coercive function over a closed set. The result follows using Corollary 2, which ensures uniqueness of the solution of (2).

Remark 5 Some assumptions are also required to prove the existence of a solution which minimizes the Gibbs energy. Here, these assumptions are directly related to the totals $T$.

We denote by $\mathcal{T}$ the set of totals $T$ such that the problem has a solution. This set is then easy to define when all the stoichiometric coefficients are positive.

Corollary 3 Assume that $S \geq 0$ and the matrix $E$ is of rank $N_{p}$ with $E \geq 0$. Then, the geochemical model (2) has a solution if and only if $T>0: \mathcal{T}=\{T \in$ $\left.\mathbb{R}^{N_{c}}, T>0\right\}$

Proof The result follows from Corollary1 and Theorem 2.

However, in the general case, it seems quite challenging to find the set $\mathcal{T}$, as illustrated by the following example. 


\subsection{Example with two minerals: calcite and gypsum}

We consider here an example where one of the stoichiometric coefficient is negative. The two reactions involve two minerals, calcite $\mathrm{CaCO}_{3}$ and gypsum $\mathrm{CaSO}_{4}$, with three aqueous components, hydrogen ion $\mathrm{H}^{+}$, calcium ion $\mathrm{Ca}^{2+}$ and sulfate ion $\mathrm{SO}_{4}^{2-}$. We simplify the mathematical model with the following assumptions: the activity of carbon dioxide $\mathrm{CO}_{2}$ is equal to 1 , and the solubility product of calcite is equal to 1 .

\begin{tabular}{|l|l|l|}
\hline Mineral & Reaction & $\kappa_{p}$ \\
\hline $\mathrm{CaCO}_{3}$ & $\mathrm{CaCO}_{3}+2 \mathrm{H}^{+} \rightleftarrows \mathrm{Ca}^{2+}+\mathrm{CO}_{2}+\mathrm{H}_{2} \mathrm{O}$ & 1 \\
\hline $\mathrm{CaSO}_{4}$ & $\mathrm{CaSO}_{4} \rightleftarrows \mathrm{Ca}^{2+}+\mathrm{SO}_{4}^{2-}$ & $410^{-5}$ \\
\hline
\end{tabular}

The stoichiometric matrix $E$ is given by

$$
E=\left(\begin{array}{ccc}
-2 & 1 & 0 \\
0 & 1 & 1
\end{array}\right)
$$

which is of rank $N_{p}$.

The following result provides necessary and sufficient conditions on $T$ for the existence of a solution.

Proposition 6 The set $\mathcal{T}$ is given by

$\mathcal{T}=\left\{\left(T_{1}, T_{2}, T_{3}\right) \in \mathbb{R}^{3}, T_{2}>0, T_{3}>0, T_{1}+2 T_{2}>0\right\}$.

Proof Mass balance equations and positivity constraints imply the necessary conditions $T_{2}>0, T_{3}>0$ and $T_{1}+2 T_{2}>0$.

Now, we show that they are sufficient.

Recall that $f(x)=\exp \left(x_{1}\right)+\exp \left(x_{2}\right)+\exp \left(x_{3}\right)-$ $T_{1} x_{1}-T_{2} x_{2}-T_{3} x_{3}$, and that $g_{1}(x)=-2 x_{1}+x_{2}-$ $\log \left(\kappa_{1}\right), g_{2}(x)=x_{2}+x_{3}-\log \left(\kappa_{2}\right)$.

For $k=2$ and $k=3$, the functions $\exp \left(x_{k}\right)-T_{k} x_{k}$ are coercive since $T_{k}>0$.

If $x_{1}$ is finite or $x_{1} \rightarrow+\infty$, then $\lim _{\left|x_{2}\right| \rightarrow+\infty} f(x)=$ $+\infty$.

Now, since $g_{1}(x) \leq 0$ we have $-x_{2} \geq-2 x_{1}-\log \left(\kappa_{1}\right)$ thus, since $T_{2}>0$, also $-T_{2} x_{2} \geq-2 T_{2} x_{1}-T_{2} \log \left(\kappa_{1}\right)$ and finally $f(x) \geq \exp \left(x_{1}\right)+\exp \left(x_{2}\right)+\exp \left(x_{3}\right)-T_{3} x_{3}-$ $\left(T_{1}+2 T_{2}\right) x_{1}-T_{2} \log \left(\kappa_{1}\right)$.

Since $T_{1}+2 T_{2}>0$, for any $\left(x_{2}, x_{3}\right)$, we get $\lim _{x_{1} \rightarrow-\infty} f(x)=+\infty$.

We conclude that the function $f$ is coercive and that the minimization problem has a solution.

\section{Precipitation Diagram}

Throughout this section, we assume that the matrix $E$ is of full rank. Then, for any $T \in \mathcal{T}$, there is a unique solution of the chemical model, which is the speciation of the geochemical system associated with the data $T$. It gives the positive concentrations of the primary components (the unknown $c$ or $x=\log (c)$ ) and the nonnegative quantities of the minerals (the Lagrange multiplier $p$ ).

Now, we propose to characterize this speciation by the presence or lack of each mineral. Then, the aim is to find the subset of totals $T$ leading to a speciation with a given subset of precipitated minerals. We build a partition of $\mathcal{T}$ into so-called mineral states, associated to subsets of minerals. This partition defines a diagram, in the same spirit as thermodynamic diagrams $[11,13]$.

This diagram can be formally defined by using the notions of strongly active or weakly active constraints. We first give some definitions based on the optimization problem, then propose a procedure to compute the diagram using symbolic calculus.

\subsection{Definitions}

For $T \in \mathcal{T}$, we denote by $(x(T), p(T))$ the unique solution of the minimization problem (6) with the data $T$. We characterize the geochemical system by the minerals, introducing the subset $M(T) \subset\left\{1,2, \ldots, N_{p}\right\}$ of precipitated minerals defined by

$M(T)=\left\{i, p_{i}(T)>0\right\}$.

Conversely, let $I$ be a subset of $\left\{1,2, \ldots, N_{p}\right\}$ and $\bar{I}$ its complement set. We call a mineral state associated to $I$ the subset of $\mathcal{T}$ such that, for any $T$ in this subset, $M(T)=I$.

Definition 1 A mineral state associated to a subset $I$ of $\left\{1,2, \ldots, N_{p}\right\}$ is defined by

$$
\mathcal{M}_{I}=\{T \in \mathcal{T}: M(T)=I\},
$$

where $M(T)$ is defined by (7). In other words, the constraints with indices in the subset $I$ are strongly active:

This definition builds a partition of the space $\mathcal{T}$ into at most $2^{N_{p}}$ mineral states, which we call a precipitation diagram.

Proposition 7 The non-empty mineral states build a partition of $\mathcal{T}$. We call this partition a precipitation diagram.

Proof By definition of $M(T)$, it holds $\cup_{I} \mathcal{M}_{I}=\mathcal{T}$. Moreover, for all $I, J$ such that $\mathcal{M}_{I}$ and $\mathcal{M}_{J}$ are nonempty, then $I \neq J \Longrightarrow \mathcal{M}_{I} \cap \mathcal{M}_{J}=\emptyset$.

The mineral states are interfaced by what we call state boundaries, which are obtained by removing a 
mineral which is dissolved and still at saturation. Therefore, each mineral state is associated to at most $N_{p}$ boundaries and there are at most $N_{p} 2^{N_{p}-1}$ non-empty boundaries (since each of them is defined by one given mineral and a subset of the other $N_{p}-1$ minerals).

Definition 2 A state boundary of the mineral state associated to $I$ and $j \in I$ is defined by

$$
\mathcal{M}_{I \backslash\{j\}} \cap\left\{T \in \mathcal{T}: p_{j}(T)=g_{j}(x(T))=0\right\} .
$$

In other words, the constraint of index $j$ is weakly active and those of other indexes in $I$ are strongly active.

The intersection of boundaries is a special state where all minerals are dissolved and still at saturation. We call it the critical state.

Definition 3 The critical state is the subset $\mathcal{C}$ defined by

$\mathcal{C}=\left\{T \in \mathcal{T}: \forall i \in\left\{1,2, \ldots, N_{p}\right\}, p_{i}(T)=g_{i}(x(T))=0\right\}$.

In other words, all constraints of the critical state are weakly active.

4.2 Example with two salts: sodium chloride and potassium chloride

Let us illustrate these definitions by the example with two salts of Section 2.3. Here, all the totals must be strictly positive to ensure a unique solution.

We further assume that the species satisfy electrical neutrality. Using the charge of each ion, the balance equation can be written $c_{1}+c_{2}-c_{3}=0$, thus $c_{3}=c_{1}+c_{2}$ can be eliminated from the equations.

By combining the mass balance equations, we also get the property $T_{1}+T_{2}-T_{3}=0$, thus we can restrict the study to only two parameters $T_{1}, T_{2}$.

The $2 \mathrm{D}$ precipitation diagram, with $T_{1}>0$ and $T_{2}>0$, is shown in Figure 1.

The space is divided into four states, with four boundaries. The critical set is the point $\left(T_{1, c} T_{2, c}\right)$ :

$$
\begin{aligned}
& T_{1, c}=\kappa_{1} / \sqrt{\kappa_{1}+\kappa_{2}}, \\
& T_{2, c}=\kappa_{2} / \sqrt{\kappa_{1}+\kappa_{2}} .
\end{aligned}
$$

The four state boundaries are:

$$
\begin{aligned}
& T_{1}=T_{1, c}, \\
& T_{2}=T_{2, c}, \\
& T_{1}\left(T_{1}+T_{2}\right)=\kappa_{1}, \\
& T_{2}\left(T_{1}+T_{2}\right)=\kappa_{2} .
\end{aligned}
$$

Here, it is easy to compute by hand the solutions and to characterize the diagram. However, for more complex geochemical systems, the task becomes tricky.

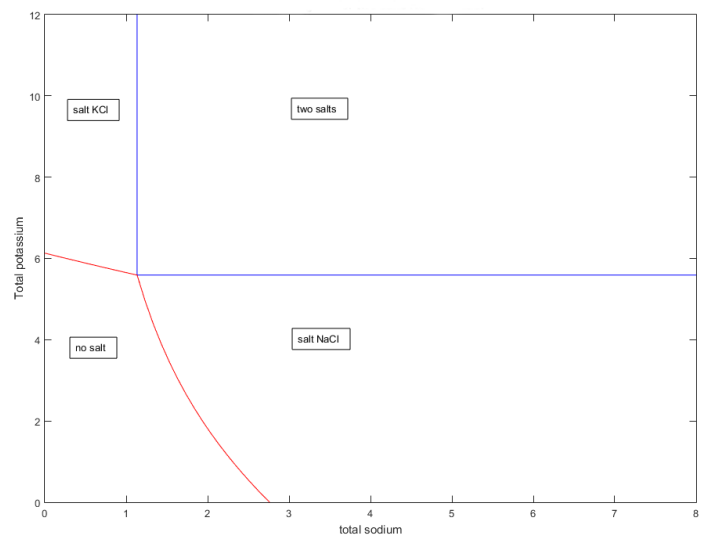

Fig. 1: Precipitation diagram with the two salts $\mathrm{NaCl}, \mathrm{KCl}$.

\subsection{Computing a precipitation diagram}

We propose to follow a combinatorial approach exploring the $2^{N_{p}}$ possible mineral states and using symbolic computations. This framework is tractable for a moderate number of minerals, say a dozen. A by-product of this procedure will be to get a symbolic expression of the solution $(c, p)$, but the main objective is to build the precipitation diagram.

The combinatorial approach should be more robust than fixing a mesh-grid on the domain of $T$ and repeatedly computing numerically the mineral state. Indeed, as can be seen in Figure 1, it might be easy to miss a phase if the mesh size is not large enough. In Figure 3, it might also be difficult make the difference between a zone without solution and a numerical failure. On the other hand, it might become very costly to refine the mesh near the boundaries.

We come back to the non-logarithmic system (2). Because we consider integer stoichiometric coefficients, equations are polynomial equalities and inequalities, which define semi-algebraic sets. Therefore, solving means finding solutions depending on algebraic numbers, which are roots of univariate polynomials.

In order to simplify the symbolic computations, we can take advantage of some invariants, such as the charge balance described for the example with two salts.

\subsection{Invariants with conservative variables}

Since we assume that the matrix $E$ is of full rank $N_{p}$, the $Q R$ factorization of the matrix $E^{T}$ yields an orthogonal matrix $\left(Q_{1}, Q_{2}\right) \in \mathbb{R}^{N_{c} \times N_{c}}$ and a non-singular 
triangular matrix $R \in \mathbb{R}^{N_{p} \times N_{p}}$ such that

$$
E^{T}=\left(Q_{1}, Q_{2}\right)\left(\begin{array}{c}
R \\
0
\end{array}\right)=Q_{1} R
$$

It can be noted that $E Q_{1}=R^{T}$ and $E Q_{2}=0$. It follows that $p$ can be written as

$p(c)=R^{-1} Q_{1}^{T}\left(T-c-S^{T} \alpha(c)\right)$.

Also, the variables $Q_{2}^{T} c$ are decoupled from minerals thanks to the relation

$Q_{2}^{T} T=Q_{2}^{T}\left(c+S^{T} \alpha(c)\right)$.

Finally, these simplifications lead to a model involving only the $N_{c}$ variables $c$ :

$$
\left\{\begin{array}{l}
Q_{2}^{T}\left(c+S^{T} \alpha(c)\right)=Q_{2}^{T} T, \\
p(c)^{T}\left(\kappa_{p}-\gamma(c)\right)=0 \\
c>0, p(c) \geq 0, \kappa_{p} \geq \gamma(c) .
\end{array}\right.
$$

The quantities $Q_{2}^{T} c$ are called conservative variables, because they are often related to some balance equation. In many cases, these equations can be used to eliminate some unknowns, in order to simplify the precipitation diagram. A similar transformation was used in [21] to simplify the system and prove strict convexity of the Gibbs energy.

An example is the charge balance, when the primary components are ions. The corresponding vector $q$ in $Q_{2}$ represents the charges of these ions. In order to ensure electrical neutrality, it is common to require that $q^{T} T=$ 0 . This invariant can be used to remove a parameter $T_{k}$ and to eliminate the unknown $c_{k}$.

\subsection{Symbolic computations}

Let $\mathcal{M}_{I}$ with $I \subset\left\{1,2, \ldots, N_{p}\right\}$ be a given mineral state and let $T \in \mathcal{T}$ be a parameter of the symbolic procedure. Then $T \in \mathcal{M}_{I}$ if and only if $(c(T), p(T))$ satisfies:

$$
\left\{\begin{array}{l}
Q_{2}^{T}\left(c+S^{T} \alpha(c)\right)-Q_{2}^{T} T=0 \\
\gamma_{i}(c)=\kappa_{p i}, \forall i \in I \\
p_{i}(c)=0, \forall i \in \bar{I} \\
c>0 \\
p_{i}(c)>0, \forall i \in I \\
\gamma_{i}(c) \leq \kappa_{p i}, \forall i \in \bar{I}
\end{array}\right.
$$

where $p(c)$ is given by (8).

We recall that this system can usually be simplified by using conservative variables and balance equations.

In this form, it is a set of $N_{c}$ polynomial equations with $N_{c}$ positivity constraints, and $N_{p}$ polynomial inequalities. We first solve the $N_{c}$ polynomial equations and get solutions in terms of algebraic numbers, which are function of $T$. We keep only those such that $c>0$.
Then, these computations allow to simplify the polynomial inequalities that now depend on the algebraic numbers computed in the previous step. Thus, we get an implicit description of the state boundaries in function of $T$.

Different approaches exist to obtain a description of the solutions of a set of polynomial equations. The usual way of representing this set of solutions is through a triangular system of equations, sometimes called regular chains. A different approach consider the rational univariate representation of F. Rouillier [32]. Several methods exist to compute a triangulation of a polynomial system, probably the most used one is to compute Gröbner bases [10,18]. This tool is in general useful as it also determines if the system is inconsistent and if it has a finite or an infinite number of solutions. The oldest and most well-known method to compute such a basis is the Buchberger's algorithm [6], but more recent and efficient methods were developed, for instance Faugère's F4 and F5 algorithms [17]. Computation of a Gröbner basis requires in the worst case $d^{2^{O(n)}}$ operations where $d$ is an upper bound on the degrees of the polynomials and $n$ the number of variables. Nowadays, most of the computer algebra systems contain routines to do so. So we do not get into the details of these computations.

\section{Numerical Experiments}

In this section, we show numerical results with three examples. Precipitation diagrams were computed with a code developed in Maple ${ }^{\mathrm{TM}}$, implementing the symbolic procedure described above.

These diagrams are computed only once for a given geochemical system. Nevertheless, time and memory requirements could be high, regarding the number of mineral states and boundaries. We provide CPU and memory measurements for the last example, with 64 states.

5.1 Three salts: sodium chloride, potassium chloride, and carnallite

We consider here an example with three minerals, sodium chloride $\mathrm{NaCl}$, potassium chloride $\mathrm{KCl}$ and carnallite $\mathrm{KMgCl}_{3}$, reacting with four aqueous components, sodium ion $\mathrm{Na}^{+}$, potassium ion $\mathrm{K}^{+}$, magnesium ion $\mathrm{Mg}^{2+}$ and chloride ion $\mathrm{Cl}^{-}$. The three reactions and the solubility products are given in the following table: 


\begin{tabular}{|c|c|c|}
\hline Mineral & Reaction & $\kappa_{p}$ \\
\hline $\mathrm{NaCl}$ & $\mathrm{NaCl} \rightleftarrows \mathrm{Na}^{+}+\mathrm{Cl}^{-}$ & 37.5837 \\
\hline $\mathrm{KCl}$ & $K C l \rightleftarrows K^{+}+C l^{-}$ & 7.6208 \\
\hline $\mathrm{KMgCl}_{3}$ & $\mathrm{KMgCl3} \rightleftarrows \mathrm{K}^{+}+\mathrm{Mg}^{2+}+3 \mathrm{Cl}^{-}$ & $2.1380 \mathrm{e}+04$ \\
\hline
\end{tabular}

The stoichiometric matrix $E$ of this system is given by

$$
E=\left(\begin{array}{llll}
1 & 0 & 0 & 1 \\
0 & 1 & 0 & 1 \\
0 & 1 & 1 & 3
\end{array}\right)
$$

Since the matrix $E$ is of full rank $N_{p}$ and all the coefficients are non-negative, the set of parameters $T$ with a unique solution is given by

$$
\mathcal{T}=\left\{T \in \mathbb{R}^{N_{c}}, T>0\right\}
$$

The kernel of $E$ has one vector $q$ defined by the charges of ions: $q=(1,1,2,-1)^{T}$. Thus, the charge balance is a conservative variable and electrical neutrality requires that $T_{1}+T_{2}+2 T_{3}=T_{4}$. As described above, we use electrical neutrality to remove the total $T_{4}$ and the concentration $c_{4}$ of chloride ion. The $3 \mathrm{D}$ precipitation diagram, with $T_{i}>0, i=1,2,3$, is shown in Figure 2.

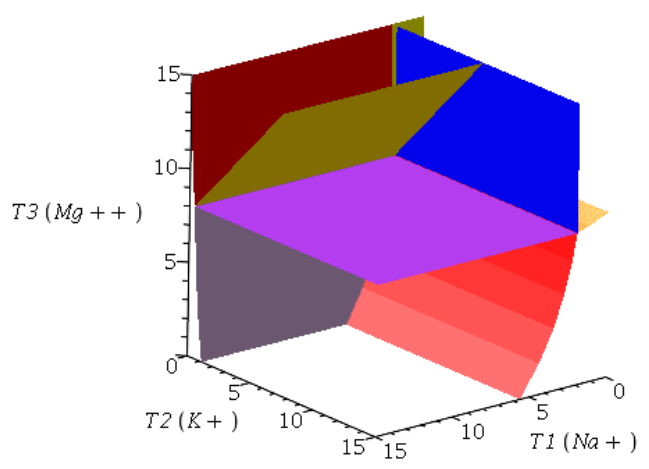

Fig. 2: Precipitation diagram with the three salts $\mathrm{NaCl}, \mathrm{KCl}$ and $\mathrm{KMgCl}_{3}$.

There is only one critical point $\left(T_{1, c}, T_{2, c}, T_{3, c}\right)$. The state with three precipitated minerals is bounded by the three hyperplanes:

$$
\begin{aligned}
& T_{1}=T_{1, c}, \\
& T_{2}=T_{2, c}+T_{3}-T_{3, c}, \\
& T_{3}=T_{3, c} .
\end{aligned}
$$

More details can be found in [28].

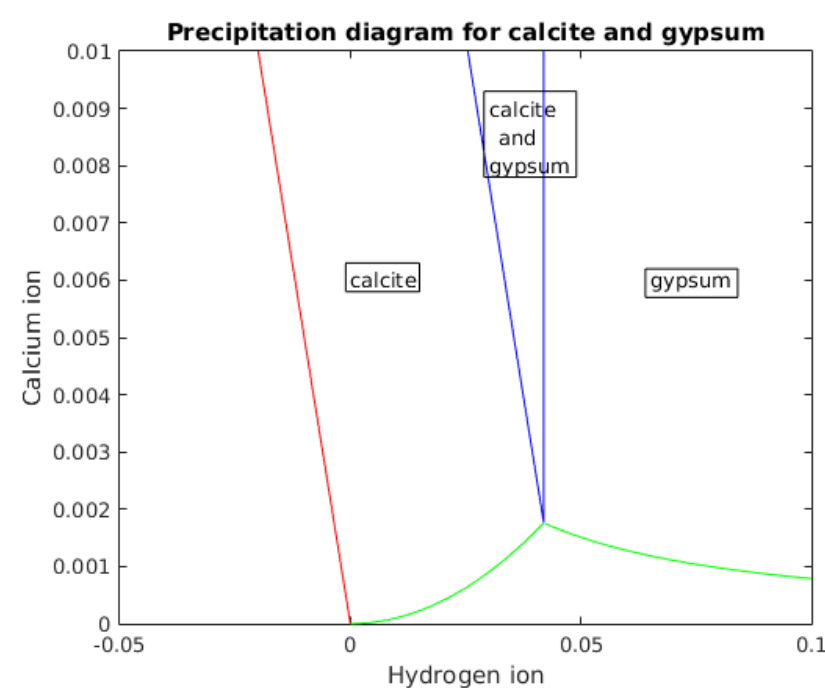

Fig. 3: Precipitation diagram with calcite and gypsum (simplified system).

\subsection{Two minerals: calcite and gypsum}

This example was already described in Section 3.4.

Here too, the matrix $Q_{2}$ has one vector which is defined by the charge of ions. Electrical neutrality allows to remove the total $T_{3}$ and the concentration $c_{3}$ of sulfate ion.

The 2D precipitation diagram is shown in Figure 3.

The critical set is the point $\left(T_{1, c} T_{2, c}\right)$ where $T_{1, c}$ is the unique positive solution of $\kappa_{1} y^{3}\left(1 / 2+\kappa_{1} y\right)-\kappa_{2}=0$ and $T_{2, c}=\kappa_{1} T_{1, c}^{2}$. The four state boundaries are given by

$$
\begin{aligned}
& T_{1}=T_{1, c}, \\
& T_{2}=T_{2, c}+1 / 2\left(T_{1, c}-T_{1}\right), \\
& T_{2}=\kappa_{1} T_{1}^{2}, \\
& T_{2}\left(1 / 2 T_{1}+T_{2}\right)=\kappa_{2} .
\end{aligned}
$$

\subsection{Acid mine drain}

We now give an example coming from the literature of acid mine drain, [29]. An acid mine drain (AMD) is an acid mineral solution which appears in some kind of mines or in the context of waste storage. The presence of AMD leads to acidification of the water and appearance of sulphuric acid $\left(\mathrm{H}_{2} \mathrm{SO}_{4}\right)$. Figure 4 gives the various species in the initial porous media. We are interested in the precipitation-dissolution reactions of Figure 5 . The solubility products come from the database MINTEQ/A2.

In order to simplify the chemistry, we consider only the minerals and the primary species. So, we get a stoichiometric matrix $E$ with three independent systems, 
Table 1

Initial background, as well as AMD source and non-source (natural recharge water) concentrations at surface for the conceptual model based on the tailings source at the Nickel Rim mine, Ontario Canada (Walter et al., 1994b).

\begin{tabular}{llll}
\hline Component & $\begin{array}{l}\text { Initial background } \\
\text { conc. }{ }^{2}(\mathrm{~mol} / \mathrm{L})\end{array}$ & $\begin{array}{l}\text { Source conc. } \\
\text { (mol/L) }\end{array}$ & $\begin{array}{l}\text { Non-source } \\
\text { conc. (mol/L) }\end{array}$ \\
\hline $\mathrm{Ca}^{2+}$ & $5.2 \mathrm{e}-03$ & $1.08 \mathrm{e}-02$ & $6.9 \mathrm{e}-03$ \\
$\mathrm{Mg}^{2+}$ & $1.5 \mathrm{e}-03$ & $9.69 \mathrm{e}-04$ & $1.9 \mathrm{e}-03$ \\
$\mathrm{Na}^{+}$ & $1.3 \mathrm{e}-03$ & $1.39 \mathrm{e}-03$ & $1.3 \mathrm{e}-03$ \\
$\mathrm{~K}^{+}$ & $6.6 \mathrm{e}-05$ & $8.14 \mathrm{e}-04$ & $6.7 \mathrm{e}-05$ \\
$\mathrm{Cl}^{-}$ & $1.0 \mathrm{e}-03$ & $1.58 \mathrm{e}-02$ & $1.0 \mathrm{e}-03$ \\
$\mathrm{CO}_{3}^{2-}$ & $2.4 \mathrm{e}-06$ & $4.92 \mathrm{e}-04$ & $3.9 \mathrm{e}-03$ \\
$\mathrm{SO}_{4}^{2-}$ & $5.3 \mathrm{e}-03$ & $5.00 \mathrm{e}-02$ & $7.5 \mathrm{e}-03$ \\
$\mathrm{Mn}^{2+}$ & $2.9 \mathrm{e}-05$ & $7.84 \mathrm{e}-03$ & $4.7 \mathrm{e}-05$ \\
$\mathrm{H}_{4} S^{2} \mathrm{Si}_{4}^{0}$ & $1.9 \mathrm{e}-03$ & $2.08 \mathrm{e}-03$ & $1.9 \mathrm{e}-03$ \\
$\mathrm{Fe}^{2+}$ & $3.6 \mathrm{e}-05$ & $3.06 \mathrm{e}-02$ & $5.4 \mathrm{e}-05$ \\
$\mathrm{Fe}^{3+}$ & $8.7 \mathrm{e}-17$ & $2.00 \mathrm{e}-07$ & $2.3 \mathrm{e}-08$ \\
$\mathrm{Al}^{3+}$ & $6.6 \mathrm{e}-13$ & $4.30 \mathrm{e}-03$ & $1.3 \mathrm{e}-07$ \\
$\mathrm{pH}^{3+}$ & 7.2 & 4 & 7.0 \\
\hline${ }^{\mathrm{a}}$ Small differences with Walter et al. (1994b) due to equilibration with modified \\
background mineralogy.
\end{tabular}
background mineralogy.

Fig. 4: Chemical species in the Acid Mine Drain test case from [29].

\begin{tabular}{|c|c|c|}
\hline Mineral & Reaction & $\log K_{k}$ \\
\hline Calcite & $\mathrm{CaCO}_{3} \Leftrightarrow \mathrm{Ca}^{2+}+\mathrm{CO}_{3}^{2-}$ & -8.5 \\
\hline Siderite & $\mathrm{FeCO}_{3} \Leftrightarrow \mathrm{Fe}^{2+}+\mathrm{CO}_{3}^{2-}$ & -10.5 \\
\hline Gibbsite & $\mathrm{Al}(\mathrm{OH})_{3}+3 \mathrm{H}^{+} \Leftrightarrow \mathrm{Al}^{3+}+3 \mathrm{H}_{2} \mathrm{O}$ & 8.1 \\
\hline Gypsum & $\mathrm{CaSO}_{4} \cdot 2 \mathrm{H}_{2} \mathrm{O} \Leftrightarrow \mathrm{Ca}^{2+}+\mathrm{SO}_{4}^{2-}+2 \mathrm{H}_{2} \mathrm{O}$ & -4.6 \\
\hline Ferrihydrite & $\mathrm{Fe}(\mathrm{OH})_{3}+3 \mathrm{H}^{+} \Leftrightarrow \mathrm{Fe}^{3+}+3 \mathrm{H}_{2} \mathrm{O}$ & 4.9 \\
\hline Quartz & $\mathrm{SiO}_{2}(\mathrm{am})+2 \mathrm{H}_{2} \mathrm{O} \Leftrightarrow 3 \mathrm{H}_{4} \mathrm{SiO}_{4}$ & -2.7 \\
\hline
\end{tabular}

Fig. 5: Mineral reactions and constants in the Acid Mine Drain test case from [29].

\begin{tabular}{|c|c|c|c|c|c|c|c|}
\hline$c_{\mathrm{H}_{4} \mathrm{SiO}_{4}}$ & $c_{H^{+}}$ & $c_{F e^{3+}}$ & $c_{A l^{3+}}$ & $c_{C a^{2+}}$ & $c_{S^{2-}}$ & $c_{F e^{2+}}$ & $c_{\mathrm{CO}_{3}^{2-}}$ \\
\hline 3 & 0 & 0 & 0 & 0 & 0 & 0 & 0 \\
\hline 0 & -3 & 0 & 1 & 0 & 0 & 0 & 0 \\
\hline 0 & -3 & 1 & 0 & 0 & 0 & 0 & 0 \\
\hline 0 & 0 & 0 & 0 & 1 & 0 & 0 & 1 \\
\hline 0 & 0 & 0 & 0 & 0 & 0 & 1 & 1 \\
\hline 0 & 0 & 0 & 0 & 1 & 1 & 0 & 0 \\
\hline
\end{tabular}

Even if the polynomial equations possess a favorable structure, there are still a high number of states $\left(2^{6}=64\right)$ to explore. In the Table below, we provide the time and memory required for the different steps of computations.

\begin{tabular}{|l|c|c|}
\hline Steps & Time (in sec.) & Memory (in MB) \\
\hline \hline Loading function library & 0.96 & 33.93 \\
\hline $\begin{array}{l}\text { Generation of the reduced } \\
\text { chemical system }\end{array}$ & $<1$ & $<1$ \\
\hline Computation of every phase & 2.14 & 16.00 \\
\hline Total & 3.18 & 49.93 \\
\hline
\end{tabular}

We observe that our symbolic software was able to compute the precipitation diagram quite quickly, with low memory requirements. The time and memory required to load the library of functions used in the computation are here to make a comparison point. Since the generation of the reduced chemical system involves only very simple operations, it requires almost no time and memory. Computing each mineral state with its boundaries out of 64 subsets is the most expensive step, as expected.

\section{Conclusion and Perspectives}

The study presented in this article shows some of the main characteristics of a geochemical system, considering a diluted solution with aqueous and precipitationdissolution reactions. We introduced a new minimization problem, with a strictly convex objective function. It was then straightforward to show that under mild classical assumptions the geochemical system has at most one solution. We also discussed existence of this solution with conditions on the totals in mass balance equations, which are here parameters of the model. We defined a set of totals such that the geochemical system has a unique solution.

Then, we introduced a precipitation diagram which provides a partition of this set into the various possible states of mineral reactions. We gave some characterization of this diagram and described a symbolic procedure to compute it, based on the polynomial structure of the problem. Numerical results using a code developed in Maple illustrated some examples of precipitation diagram and showed that the whole computation is actually reasonable in time and memory.

Reactive transport modeling is one of the main applications using the geochemical model. It is clear that the computation of a precipitation diagram cannot be included in a full code for this application since in this case the non-linear equation has to be solved millions of time. However, it can be very useful in describing the chemical reactions and so it can be used to find a good initial point for a numerical code or to compute the exact solution in a verification process. These extensions to reactive transport and applications of the precipitation diagram are left for further research.

Our results currently apply to diluted solutions, with a simplified model of activities. An important perspective is to extend them in a more general context, where activities are functions of the quantities of species.

\section{References}

1. I. Ben Gharbia and J. Jaffré. Gas phase appearance and disappearance as a problem with complementarity con- 
straints. Mathematics and Computers in Simulation, 99:2836,2014

2. C. M. Bethke. Geochemical Reaction Modeling : Concepts and Applications. Oxford University Press US, 1996.

3. C. M. Bethke. Geochemical and biogeochemical reaction modeling. Cambridge University Press, 2007.

4. J.-F. Bonnans, J. C. Gilbert, C. Lemaréchal, and C. A. Sagastizábal. Numerical optimization: theoretical and practical aspects. Springer Science \& Business Media, 2006.

5. F. Brunner. Multiphase multicomponent flow in porous media with general reactions: efficient problem formulations, conservative discretizations, and convergence analysis. $\mathrm{PhD}$ thesis, Universität Erlangen-Nürnberg, 2015.

6. B. Buchberger. Ein algorithmus zum auffinden der basiselemente des restklassenrings nach einem nulldimensionalen polynomideal. Universitat Innsbruck, Austria, Ph. D. Thesis, 1965 .

7. J. Carrayrou. Looking for some reference solutions for the reactive transport benchmark of momas with specy. Computational Geoscience, 14:393-403, 2010.

8. J. Carrayrou, M. Kern, P. Knabner. Reactive transport benchmark of MoMaS. Computational Geoscience, 14:385392, 2010.

9. J. Carrayrou, J. Hoffmann, P. Knabner, S. Kräutle, C. De Dieuleveult, J. Erhel, J. Van Der Lee, V. Lagneau, K.U. Mayer, and K. TB. Macquarrie. Comparison of numerical methods for simulating strongly nonlinear and heterogeneous reactive transport problemsthe MoMaS benchmark case. Computational Geosciences, 14(3):483502,2010

10. A. M. Cohen, H. Cuypers, and H. Sterk, editors. Some tapas of computer algebra, volume 4 of Algorithms and Computation in Mathematics. Springer-Verlag, Berlin, 1999.

11. J. A. D. Connolly and K. Petrini. An automated strategy for calculation of phase diagram sections and retrieval of rock properties as a function of physical conditions. Journal of Metamorphic Geology, 20(7):697-708, 2002.

12. R. W. Cottle, J.-S. Pang, and R. E. Stone. The Linear Complementarity Problem, volume 60. SIAM, 2009.

13. C. de Capitani and K. Petrakakis. The computation of equilibrium assemblage diagrams with Theriak/Domino software. American Mineralogist, 95(7):1006-1016, 2010.

14. J. Erhel and S. Sabit. Analysis of a global reactive transport model and results for the MoMaS benchmark. Mathematics and Computers in Simulation, 137: 286-298, 2017.

15. C. de Dieuleveult, J. Erhel, and M. Kern. A global strategy for solving reactive transport equations. Journal of Computational Physics, 228, 2009.

16. F. Facchinei and J.-S. Pang. Finite-dimensional variational inequalities and complementarity problems. Springer Science \& Business Media, 2007.

17. J.-C. Faugere. A new efficient algorithm for computing Gröbner bases (F 4). Journal of pure and applied algebra, 139(1):61-88, 1999.

18. J.-C. Faugere, P. Gianni, D. Lazard, and T. Mora. Efficient computation of zero-dimensional Gröbner bases by change of ordering. Journal of Symbolic Computation, 16(4):329-344, 1993.

19. F. Georget, J. H. Prévost, and R. J. Vanderbei. A speciation solver for cement paste modeling and the semismooth Newton method. Cement and Concrete Research, $68: 139-147,2015$

20. J. Hoffmann, S. Kräutle, and P. Knabner. A parallel global-implicit 2-d solver for reactive transport problems in porous media based on a reduction scheme and its application to the momas benchmark problem. Computational Geoscience, 14:421-433, 2010.
21. J. Hoffmann, S. Kräutle, and P. Knabner. A general reduction scheme for reactive transport in porous media. Computational Geoscience, 16:1081-1099, 2012.

22. S. Kräutle. The semismooth Newton method for multicomponent reactive transport with minerals. Advances in water resources, 34(1):137-151, 2011.

23. J. Kyparisis. On uniqueness of Kuhn-Tucker multipliers in nonlinear programming. Mathematical Programming, 32(2):242-246, 1985.

24. A. M. Leal, D. A. Kulik, G. Kosakowski. Computational methods for reactive transport modeling: a Gibbs energy minimization approach for multiphase equilibrium calculations. Advances in Water Resources, 88:231-240, 2016.

25. A. M. Leal, D. A. Kulik, W. R. Smith, M. O. Saar. An overview of computational methods for chemical equilibrium and kinetic calculations for geochemical and reactive transport modeling. Pure and Applied Chemistry, 89(5):597-643, 2017.

26. P. C. Lichtner. Continuum formulation of multicomponent-multiphase reactive transport. Reviews in Mineralogy and Geochemistry, 1996.

27. K. Ulrich Mayer and Kerry T. B. MacQuarrie. Solution of the momas reactive transport benchmark with min3pmodel formulation and simulation results. Computational Geoscience, 14:405-419, 2010.

28. T. Migot and J. Erhel. Analyse mathématique de modèles géochimiques. Research report, INRIA Rennes, October 2014.

29. J. Molson, M. Aubertin, and B. Bussiere. Reactive transport modelling of acid mine drainage within discretely fractured porous media: Plume evolution from a surface source zone. Environmental Modelling \& Software 38, 2012.

30. F. Morel and J. G. Hering. Principles and application of aquatic chemistry. Wiley- IEEE, 1993.

31. D. L. Parkhurst and C. A. J. Appelo. Description of input and examples for PHREEQC version 3: a computer program for speciation, batch-reaction, one-dimensional transport, and inverse geochemical calculations. Technical report, US Geological Survey, 2013.

32. F. Rouillier. Solving zero-dimensional systems through the rational univariate representation. Applicable Algebra in Engineering, Communication and Computing, 9(5):433461, May 1999.

33. F. Saaf, R. A. Tapia, S. Bryant, and M. F. Wheeler. Computing general chemical equilibria with an interior-point method, volume 1, pages 201-208. Computational Mechanics Publ, 1996.

34. F. E. Saaf. A study of reactive transport phenomena in porous media. PhD thesis, Rice University, 1997.

35. S. Sabit. Les méthodes numériques de transport réactif. PhD thesis, Université Rennes 1, 2014.

36. N. Z. Shapiro and L. S. Shapley. Mass action laws and the Gibbs free energy function. Journal of the Society for Industrial and Applied Mathematics, 13(2):353-375, 1965.

37. W. R. Smith and R. W. Missen. Chemical Reaction Equilibrium Analysis: Theory and Algorithms. WileyInterscience, 1982.

38. C. I. Steefel and K. T. B. MacQuarrie. Approaches to modeling of reactive transport in porous media. Reviews in Mineralogy and Geochemistry, 1996.

39. J. F. J. Zeleznik and S. Gordon. Calculation of complex chemical equilibria. Industrial and Engineering Chemistry, 1968. 